\title{
Pulmonary Talaromycosis: A Window into the Immunopathogenesis of an Endemic Mycosis
}

\author{
Shanti Narayanasamy $(D)$ John Dougherty $\cdot$ H. Rogier van Doorn • \\ Thuy Le (i)
}

Received: 18 December 2020/ Accepted: 7 June 2021 / Published online: 6 July 2021

(C) The Author(s) 2021

\begin{abstract}
Talaromycosis is an invasive mycosis caused by the thermally dimorphic saprophytic fungus Talaromyces marneffei $(\mathrm{Tm})$ endemic in Asia. Like other endemic mycoses, talaromycosis occurs predominantly in immunocompromised and, to a lesser extent, immunocompetent hosts. The lungs are the primary portal of entry, and pulmonary manifestations provide a window into the immunopathogenesis of talaromycosis. Failure of alveolar macrophages to destroy $\mathrm{Tm}$ results in reticuloendothelial system dissemination and multi-organ disease. Primary or secondary immune defects that reduce $\mathrm{CD} 4^{+} \mathrm{T}$ cells, INF- $\gamma$, IL-12, and IL-17 functions, such as HIV infection, anti-interferon- $\gamma$ autoantibodies, STAT-1 and STAT-3 mutations, and CD40 ligand deficiency,
\end{abstract}

Handling Editor: Darius Armstrong-James

S. Narayanasamy $(\bowtie)$ J. Dougherty $\cdot$ T. Le $(\bowtie)$

Division of Infectious Diseases and International Health, Duke University School of Medicine, Durham, NC, USA

e-mail: shanti.narayanasamy@duke.edu

T. Le

e-mail: thuy.le@duke.edu

H. R. van Doorn · T. Le

Oxford University Clinical Research Unit, Hanoi,

Vietnam

H. R. van Doorn

Centre for Tropical Medicine and Global Health, Nuffield Department of Medicine, University of Oxford, Oxford, UK highlight the central roles of Th1 and Th17 effector cells in the control of Tm infection. Both upper and lower respiratory infections can manifest as localised or disseminated disease. Upper respiratory disease appears unique to talaromycosis, presenting with oropharyngeal lesions and obstructive tracheobronchial masses. Lower respiratory disease is protean, including alveolar consolidation, solitary or multiple nodules, mediastinal lymphadenopathy, cavitary disease, and pleural effusion. Structural lung disease such as chronic obstructive pulmonary disease is an emerging risk factor in immunocompetent hosts. Mortality, up to $55 \%$, is driven by delayed or missed diagnosis. Rapid, non-culture-based diagnostics including antigen and PCR assays are shown to be superior to blood culture for diagnosis, but still require rigorous clinical validation and commercialisation. Our current understanding of acute pulmonary infections is limited by the lack of an antibody test. Such a tool is expected to unveil a larger disease burden and wider clinical spectrum of talaromycosis.

Keywords Talaromyces marneffei . Talaromycosis . Respiratory tract · Cavitation · Nodule $\cdot$ Effusion 


\section{Introduction}

Talaromycosis is an invasive mycosis caused by the thermally dimorphic fungus Talaromyces marneffei (Tm) endemic to Southeast Asia, southern China, and north-eastern India. Across the endemic region, talaromycosis is estimated to have a pooled prevalence of $3.6 \%$ (range $0.13-19.63 \%$ ) in people living with HIV/AIDS, with the greatest risk of infection in people with a CD4 cell count $<200$ cells $/ \mathrm{mm}^{3}$ (OR 12.68, 95\%CI: 9.58-16.77) [1]. Increasingly, talaromycosis has been described in non-HIV-infected people, accounting for $12.3 \%(82 / 668)$ of cases in one estimate [2]. The majority of these cases have other immunosuppressing conditions, including primary immunodeficiencies, auto-immune diseases, malignancies, and iatrogenic immunosuppression [3]. Despite antifungal therapy mortality remains unacceptably high, between 15 and 55\% [2, 4-6]. The soil-burrowing bamboo rats are the enzootic reservoir of Tm. However, human talaromycosis is not linked to bamboo rat exposure or consumption; rather, occupational exposure to crops and livestock is a risk factor [7]. Incidence increases $30-50 \%$ during the rainy months $[6,8]$, but is associated with increased humidity, rather than precipitation [9]. This suggests that humidity creates favourable conditions for fungal growth and aerosol dispersion, increasing respiratory exposure and infections in humans. This paper focuses on the immunopathogenesis and pulmonary manifestations of talaromycosis, highlighting unique clinical features, challenges, and research needs.

\section{Immunopathogenesis}

The presence of upper and lower respiratory tract diseases in humans suggests that inhalation is the dominant portal of entry in talaromycosis. Studies in wild bamboo rats have found the highest burden of disease in the lungs $(83.3 \%)$, followed by the liver (33.3\%) and spleen (33.3\%) [10]. A murine inhalation model delivering nebulised Tm conidia in a chamber to mice demonstrated that the lungs were the primary organ of infection with invasive pulmonary disease, occurring in $65 \%$ of exposed mice [11]. At the hostpathogen interface, Tm conidia adhere to bronchial epithelial cells by attaching to extra-cellular matrix laminin and are phagocytosed by pulmonary alveolar macrophages [12]. Although phagocytosis occurs, Tm evades macrophage killing by producing superoxide dismutase and catalase-peroxidase to prevent digestion by lysosomes [13]. Tm further evades the host defence through down-regulation of IL-6, a proinflammatory cytokine produced by bronchial epithelial cells [14], and through the galactomannanprotein Mp1p which effectively captures arachidonic acid and disrupts the host pro-inflammatory cascade [15]. The establishment and proliferation of infection inside macrophages enables $\mathrm{Tm}$ to disseminate through the reticuloendothelial system causing multi-organ disease.

Defects in cellular immunity and $\mathrm{CD} 4^{+}$lymphopenia are the major predisposing factors for talaromycosis, as demonstrated by the high burden of infection in individuals with advanced HIV and other $\mathrm{T}$ cell immunosuppression [3]. Development of talaromycosis among individuals with inborn errors of immunity provides insights into the immune mechanisms essential for disease control. The adult-onset immunodeficiency condition due to anti-interferon- $\gamma$ autoantibodies inhibits signal transducer and activator of transcription 1 (STAT1) phosphorylation and IL-12 production, leading to a severely compromised Th1 response [16]. In addition to fungal diseases, these individuals are at risk for mycobacterial diseases, disseminated varicella zoster, and salmonellosis [17]. Isolated STAT1 deficiency, both inherited and sporadic, has been described, as well as hyper-IgE (Job's) syndrome caused by STAT3 mutations resulting in Th-17 deficiency [18]. CD40 ligand deficiency (hyperIgM syndrome) causes down-regulation of activated $T$ cells, resulting in decreased signalling through NF$\kappa \mathrm{B}$ and reduction in IL-12 production [18]. These immune deficiencies reveal the central roles of IL-12 and INF- $\gamma$ interaction for macrophage activation and Th17 effector cells in host control of Tm.

\section{Pulmonary Manifestations}

Both immunocompetent and immunocompromised individuals can develop talaromycosis, and disease can be localised or disseminated [19]. Disseminated disease has traditionally been associated with advanced HIV infection and localised disease in non-HIV-infected hosts. However, evidence is emerging that both localised respiratory and disseminated 
disease can develop in HIV-infected and non-HIVinfected hosts and host immune function cannot be inferred from clinical presentation $[3,17,19,20]$. We will describe the pathology in the lungs based on anatomical location-upper respiratory and lower respiratory tracts-which have been associated with localised as well as disseminated disease.

\section{Upper Respiratory Tract Infection}

Uncommon among endemic fungi, upper respiratory tract infection is a unique manifestation of talaromycosis [21] and has been described in immunocompetent and immunocompromised patients [22-25]. Pharyngeal and laryngeal lesions have been identified in patients presenting with odynophagia, dysphagia, hoarseness, soft tissue masses, papules, and mucosal ulceration (Fig. 1) [25-28]. Tracheal and endobronchial lesions are often accompanied by cervical lymphadenopathy, pulmonary infiltrates, or post-obstructive pneumonia [29, 30]. Although uncommon, clinical manifestations can be dramatic with structural collapse of the large airways or tracheal stenosis causing airway obstruction [29, 31]. Tracheobronchial infections can lead to long-term sequelae, requiring tracheostomy and reconstructive surgery [24]. Biopsy for histopathology and cultures for both fungi and mycobacteria are critical to differentiate talaromycosis from tuberculosis, head and neck cancer, lymphoma, or Kaposi's sarcoma.

\section{Lower Respiratory Tract Infection}

Lower respiratory tract infections are protean, encompassing alveolar consolidation, solitary or multiple nodules, mediastinal lymphadenopathy, pulmonary mycetoma, cavitary disease, and pleural effusion (Fig. 2) [22, 32, 33]. Radiological characteristics are similarly diverse, including thick- and thin-walled cavitations, patchy consolidation, ground glass changes, reticular nodular changes, and hilar and mediastinal lymphadenopathy $[20,34,35]$. Cavitary disease had been thought to predominate in non-HIVinfected patients; however, a study found that talaromycosis was the most common cause of cavitary lung lesions in 81 patients with HIV/AIDS (23.5\%), followed by cryptococcosis $(13.6 \%)$ and tuberculosis $(13.6 \%)$ [36]. The diagnosis of pulmonary diseases in patients with advanced HIV/AIDS is challenging due to the frequency and clinical mimicry of other infections, including Pneumocystis jiroveci pneumonia, tuberculosis, cryptococcosis and histoplasmosis, posing significant diagnostic and therapeutic dilemmas.

Pleural effusion is an under-recognised manifestation of talaromycosis. In a non-HIV-infected cohort

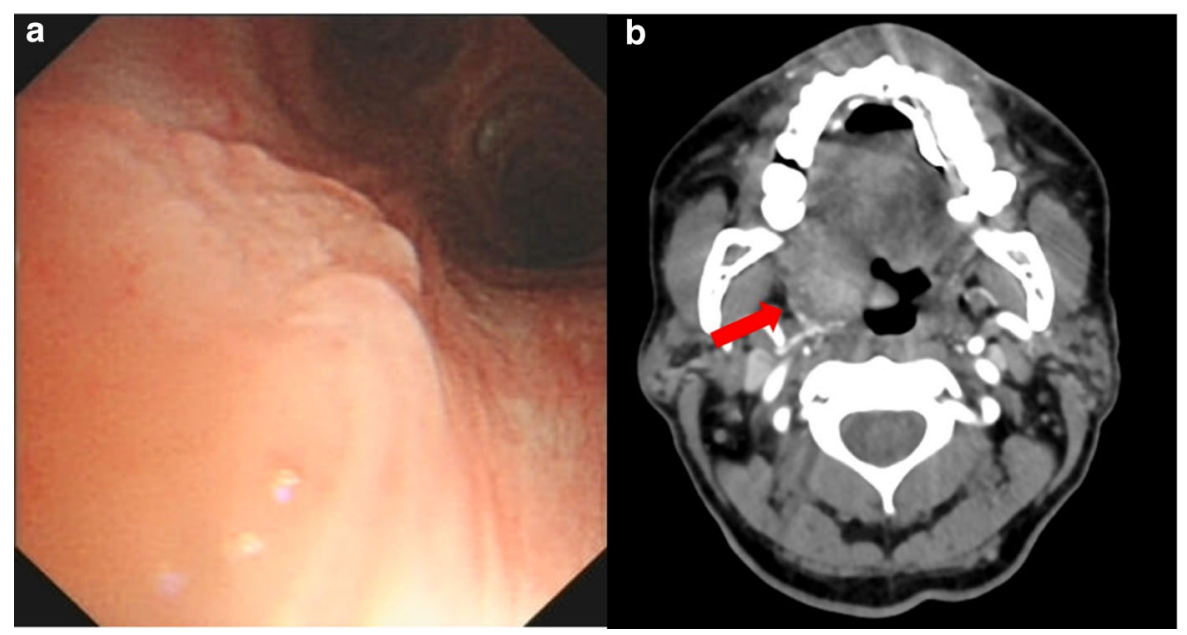

Fig. 1 Upper respiratory tract manifestations of talaromycosis. a Bronchoscopy demonstrating irregular bronchial mucosal surface, in a 34-year-old immunocompromised female with a STAT3 mutation. Tm was grown from biopsied tissue [59]. b Computed tomography (CT) angiogram of the neck demonstrating an ill-defined mass along the right lateral aspect of the hypopharynx involving the base of the tongue, right lingual tonsil, and right vallecula extending along the right palatine tonsil and into the pharyngeal space, in a 63 year-old man with HIV [27] 


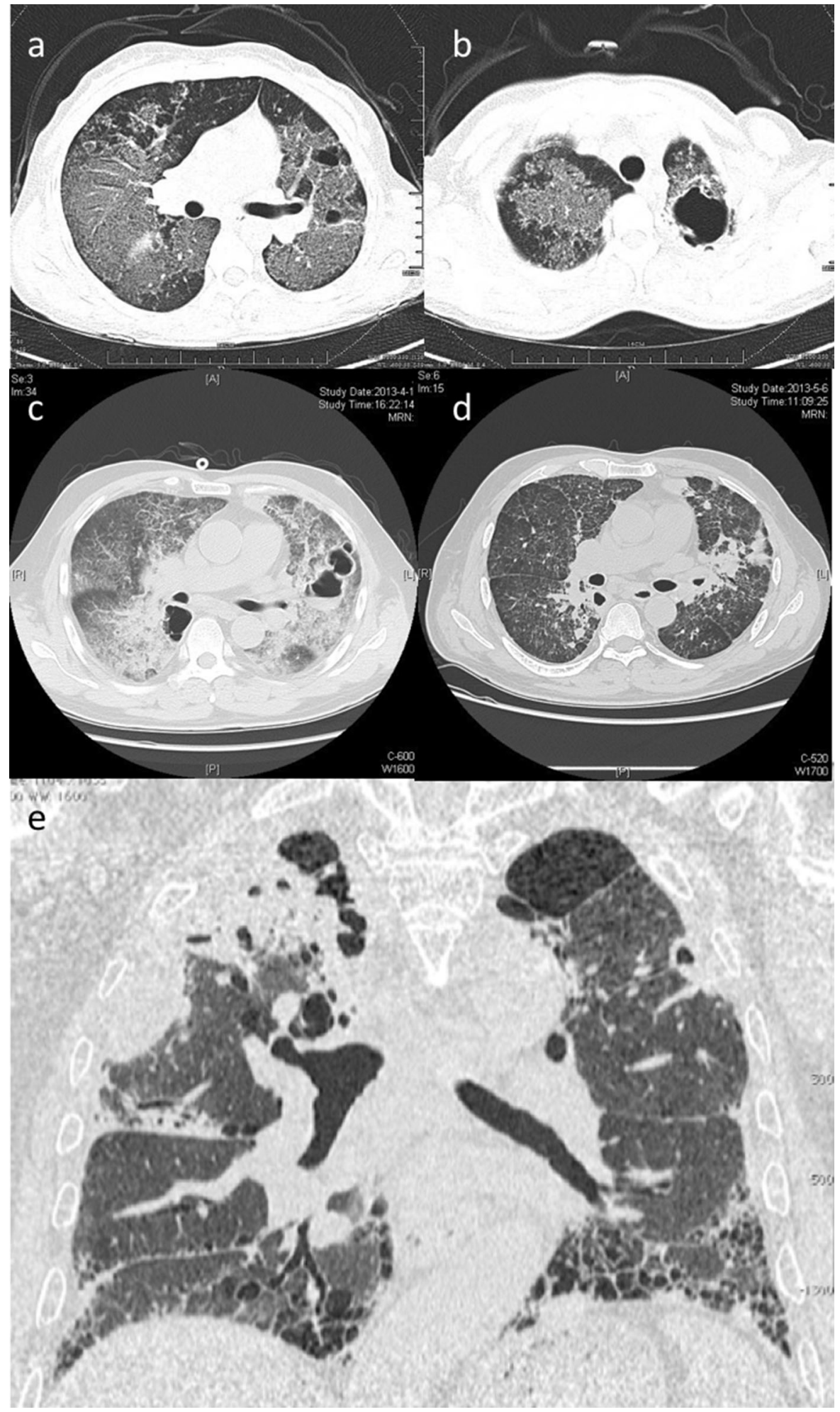


४Fig. 2 Lower respiratory tract manifestations of talaromycosis. a \& b: CT Chest demonstrating extensive bilateral ground glass changes and multiple bullae, in a 34-year-old immunocompromised female with a STAT3 mutation [59]. c: CT Chest with diffuse interstitial infiltrates and multiple cavitary lesions in a 57-year-old man following steroid use [60]. CT Chest $\mathbf{d}$ following treatment, demonstrating marked regression of interstitial and cavitary lung disease. e CT Chest demonstrating right upper lobe infiltration with Tm, bibasilar subpleural reticular changes, traction bronchiectasis and honeycombing in a 71-year-old, immunocompetent patient with idiopathic pulmonary fibrosis [61]

from China, 42/61 (69\%) had pleural effusions; all cases were initially misdiagnosed as tuberculosis [37]. Compared to tuberculous pleural effusions, talaromycosis effusions have a lower protein content and lymphocyte count, and a higher neutrophil count. The diagnosis is often made by pleural biopsy and culture, and thoracoscopy demonstrates fibrous pleural adhesions and multiple pleural nodules [37]. Talaromycosis may be unique among endemic mycoses and other opportunistic mycoses (i.e. aspergillosis, mucormycosis) in causing pleural effusions and should be considered in the differential diagnosis in a susceptible host [22].

\section{Structural Lung Disease in Immunocompetent Hosts}

Structural lung disease is increasingly recognised as a risk factor for talaromycosis among immunocompetent hosts. Case reports describe lung malignancy, both primary and metastatic, leading to talaromycosis post-obstructive pneumonia or diffuse infiltrates. These cases have been diagnosed prior to any immunosuppression, at cancer presentation [38-40]. Talaromycosis has also been described in immunocompetent patients with chronic obstructive pulmonary disease and disruption of lung architecture from other cavitary diseases [41, 42]. Cases of pneumonia and disseminated disease have been reported in immunocompetent hosts [20, 43, 44], suggesting that talaromycosis may be a more common cause of community-acquired pneumonia than currently recognised. At present, incidence of non-severe disease in immunocompetent hosts is not known, as diagnostic capabilities for talaromycosis are largely restricted to culture, which is poorly sensitive for diagnosis [45].

\section{Challenges and Advances in Diagnosis and Treatment}

Diagnostic delay is the most critical barrier to reducing morbidity and mortality. Aside from the characteristic central-umbilicated skin lesions seen in $50-70 \%$ of disseminated disease, talaromycosis has no pathognomonic clinical or radiological syndrome. It shares an endemic area and clinical presentations with tuberculosis, non-tuberculous mycobacteria, histoplasmosis, cryptococcosis, rhodococcus, and melioidosis, often resulting in misdiagnosis as one of these infections, delays in treatment, dissemination of infection, and poor patient outcomes [22, 46, 47]. Diagnostic delay is associated with higher mortality, regardless of immunosuppressive status [2, 22]. Increased access to HIV and antifungal therapy have not improved Tm mortality, suggesting that diagnostic delay remains a significant barrier to survival [4].

A preliminary diagnosis of talaromycosis can be made on microscopic examination of skin scrapings, bone marrow and lymph node aspirate, or tissue section [48]. A definitive diagnosis can be made with histopathologic examination or culture. Bone marrow culture has the highest yield (100\%), followed by skin (90\%), and blood (70\%) [6, 49].

The commercial assay for serum Aspergillus galactomannan was investigated in HIV-positive patients with talaromycosis and found a sensitivity of $95.8 \%(23 / 24)$ and specificity of $90.9 \%(30 / 33)$ at an optical density cut-off index of 1.0 [50]. A more recent study of the same assay found a lower sensitivity of $80.56 \%(29 / 36)$ and a specificity of $90 \%(27 / 30)$ at a lower optical density cut-off index of 0.5 [51]. The $\beta$-D-glucan assay has been used in some case reports [52]. These antigen-detection assays may have a role as a screening tool for patients at high risk for talaromycosis and other invasive fungal infections in high burden regions due to their cross-reactivity with other endemic fungal pathogens [52].

Several promising non-culture-based diagnostics specific for talaromycosis are in development. The galactomannanprotein Mp1p is a sensitive and highly specific target for antigen detection by enzyme immunoassay (EIA) [53]. Compared to blood culture, 
the Mp1p EIA improved time-to-diagnosis from $6.6 \pm 3.0$ days to 6 hours, and improved sensitivity from $72.8 \%(271 / 372)$ to $86.3 \%$ (321/372); higher sensitivity was demonstrated with combined plasma and urine testing [45]. Real-time quantitative PCR assays show promise in those with fungemia (sensitivity $100 \%$, [20/20]) and without fungemia (68.75\%, [11/16]) [51], and recent development of the 4D1 monoclonal antibody against the yeast phase of Tm has shown high specificity and sensitivity in an inhibitoryEIA and an immunochromatographic test $[54,55]$.

There are a lack of studies to guide treatment specifically for pulmonary talaromycosis. Evidence for talaromycosis treatment has largely come from studies in HIV-infected patients with disseminated disease. Induction therapy with amphotericin B for two weeks followed by prolonged periods of consolidation and maintenance therapy with itraconazole is highly effective when combined with suppressive HIV therapy [56]. Optimal treatment for localised disease of the respiratory tract has not been defined. Singleagent itraconazole, voriconazole, and amphotericin B have been used [22, 57]. In a report of two non-HIVinfected patients with disease involving the trachea, relapse occurred in one patient at three months despite systemic antifungal therapy and surgery, whereas cure at one year was observed in the patient who received systemic and adjunctive inhaled amphotericin B for two months, suggesting a role for inhaled amphotericin $\mathrm{B}$ in treatment of localised respiratory tract disease [58]. Optimal duration of therapy for nonHIV-infected patients remains to be defined. At present, treatment choice and duration are individualised based on the underlying immunodeficiency state, antifungal and immunosuppressive drug-drug interactions, and the expected timeframe of immune dysfunction.

\section{Conclusions}

Pulmonary talaromycosis is clinically diverse and is likely more common than currently recognised. Unlike other endemic mycoses, talaromycosis has a propensity to cause nodules and masses in the upper respiratory tract, and cause pleural effusions. Lower respiratory disease is increasingly recognised in immunocompetent hosts with structural lung diseases. Delay in diagnosis is the most challenging clinical problem. Mortality remains unacceptably high. Research should focus on improving disease detection through development and rigorous clinical validation of antigen detection and PCR-based assays. Development of antibody testing is needed to diagnose acute pulmonary infections and to enable accurate estimates of disease burden and geographical risk regions. These tools are expected to reveal a higher incidence and a wider clinical spectrum of talaromycosis than currently recognised.

Acknowledgements We acknowledge the contributions of authors who provided permission for the images included in this manuscript: Dr. M. Mizusawa, Dr. H. Furusawa, Dr. Jian Ye, and Dr. H. R. Li.

Authors' contributions SN and TL conceived and designed the study. SN performed the literature search, data analysis, and obtained permission for the use of the figures. SN and TL wrote the first draft of the manuscript. JD and HRD contributed to its intellectual content. All authors revised and approved the final manuscript.

Funding This work is funded by the Duke Center for AIDS Research Pilot Award (NIH 5P30AI064518 to Narayanasamy and Le), the Wellcome Trust (van Doorn, 106680/Z/14/Z), and the National Institute of Allergy and Infectious Diseases (NIH R0101AI143409 to Le and van Doorn).

\section{Declarations}

Conflict of interest Le has received investigator-initiated research funding from Gilead Sciences outside of the submitted work. Other authors have no relevant financial or non-financial interests to disclose.

Open Access This article is licensed under a Creative Commons Attribution 4.0 International License, which permits use, sharing, adaptation, distribution and reproduction in any medium or format, as long as you give appropriate credit to the original author(s) and the source, provide a link to the Creative Commons licence, and indicate if changes were made. The images or other third party material in this article are included in the article's Creative Commons licence, unless indicated otherwise in a credit line to the material. If material is not included in the article's Creative Commons licence and your intended use is not permitted by statutory regulation or exceeds the permitted use, you will need to obtain permission directly from the copyright holder. To view a copy of this licence, visit http://creativecommons.org/licenses/by/4.0/.

\section{References}

1. Qin Y, Huang X, Chen H, Liu X, Li Y, Hou J, et al. Burden Of talaromyces marneffei infection in people living with Hiv/Aids in Asia during art Era: a systematic review and 
meta-analysis. Bmc Infect Dis. 2020;20(1):551. https://doi. org/10.1186/S12879-020-05260-8.

2. Hu Y, Zhang J, Li X, Yang Y, Zhang Y, Ma J, et al. Penicillium marneffei infection: an emerging disease in Mainland China. Mycopathologia. 2013;175(1-2):57-67. https:// doi.org/10.1007/S11046-012-9577-0.

3. Jf C, Sk L, Ky Y, Pc W. Talaromyces (penicillium) marneffei infection in non-Hiv-infected patients. Emerg Microbes Infect. 2016;5(3):E19. https://doi.org/10.1038/ Emi.2016.18.

4. Jiang J, Meng S, Huang S, Ruan Y, Lu X, Jz Li, Al Et. Effects of talaromyces marneffei infection on mortality of Hiv/Aids patients in southern China: a retrospective cohort study. Clin Microbiol Infect. 2019;25(2):233-41. https:// doi.org/10.1016/J.Cmi.2018.04.018.

5. Pp L, Kw C, Tl L, Ho Mh, Xy C, Li Ch, et al. Penicilliosis in children without Hiv infection-are they immunodeficient? Clin Infect Dis. 2012;54(2):E8-19. https://doi.org/10.1093/ Cid/Cir754.

6. Le T, Wolbers M, Chi Nh, Vm Quang, Nt Chinh, Np Lan, et al. Epidemiology, seasonality, and predictors of outcome of Aids-associated penicillium marneffei infection in Ho Chi Minh city. Viet Nam Clin Infect Dis. 2011;52(7):945-52. https://doi.org/10.1093/Cid/Cir028.

7. Chariyalertsak S, Sirisanthana T, Supparatpinyo K, Praparattanapan J, Ke Nelson. Case-control study of risk factors for penicillium marneffei infection in human immunodeficiency virus-infected patients in Northern Thailand. Clin Infect Dis. 1997;24(6):1080-6. https://doi. org/10.1086/513649.

8. Chariyalertsak S, Sirisanthana T, Supparatpinyo K, Ke Nelson. Seasonal variation of disseminated penicillium marneffei infections in northern Thailand: a clue to the reservoir? J Infect Dis. 1996;173(6):1490-3. https://doi.org/ 10.1093/Infdis/173.6.1490.

9. Pl Bulterys, Le T, Vm Quang, Ke Nelson, Jo Lloyd-Smith. Environmental predictors and incubation period of aidsassociated penicillium marneffei infection in Ho Chi Minh city. Vietnam Clin Infect Dis. 2013;56(9):1273-9. https:// doi.org/10.1093/Cid/Cit058.

10. Ajello L, Aa Padhye, Sukroongreung S, Nilakul Ch, Tantimavanic S. Occurrence of penicillium marneffei infections among wild bamboo rats in Thailand. Mycopathologia. 1995;131(1):1-8. https://doi.org/10.1007/Bf01103897.

11. Liu Y, Huang X, Yi X, He Y, Mylonakis E, Xi L. Detection of talaromyces marneffei from fresh tissue of an inhalational murine pulmonary model using nested pcr. PLoS ONE. 2016;11(2):E0149634. https://doi.org/10.1371/Journal. Pone.0149634.

12. Ellett F, Pazhakh V, Pase L, El Benard, Weerasinghe H, Azabdaftari D, et al. macrophages protect talaromyces marneffei conidia from myeloperoxidase-dependent neutrophil fungicidal activity during infection establishment in vivo. Plos Pathog. 2018;14(6):E1007063. https://doi.org/ 10.1371/Journal.Ppat.1007063.

13. Pongpom M, Vanittanakom P, Nimmanee P, Cooper CR, Vanittanakom N. Adaptation to macrophage killing by talaromyces marneffei. Future Sci Oa. 2017;3(3):Fso215. https://doi.org/10.4155/Fsoa-2017-0032.

14. Li Y, Chen H, Li S, Li Y, Liu G, Bai J, et al. Lncssbp1 functions as a negative regulator of il-6 through interaction with hnrnpk in bronchial epithelial cells infected with talaromyces marneffei. Front Immunol. 2019;10:2977. https://doi.org/10.3389/Fimmu.2019.02977.

15. Lam Wh, Sze Kh, Ke Y, Mk Tse, Zhang H, Pcy Woo, Al Et. Talaromyces marneffei $\mathrm{mp}$ protein, a novel virulence factor, carries two arachidonic acid-binding domains to suppress inflammatory responses in hosts. Infect Immun. 2019. https://doi.org/10.1128/Iai.00679-18.

16. Sk Browne, Burbelo Pd, Chetchotisakd P, Suputtamongkol Y, Kiertiburanakul S, Pa Shaw, et al. Adult-onset immunodeficiency in Thailand and Taiwan. N Engl J Med. 2012;367(8):725-34. https://doi.org/10.1056/ Nejmoa1111160.

17. Qiu Y, Feng X, Zeng W, Zhang H, Zhang J. Immunodeficiency disease spectrum in Hiv-negative individuals with talaromycosis. J Clin Immunol. 2020. https://doi.org/10. 1007/S10875-020-00869-5.

18. Pp L, Y1 L. Cellular and molecular defects underlying invasive fungal infections-revelations from endemic mycoses. Front Immunol. 2017;8:735. https://doi.org/10. 3389/Fimmu.2017.00735.

19. Cao C, Xi L, Chaturvedi V. Talaromycosis (penicilliosis) due to talaromyces (penicillium) marneffei: insights into the clinical trends of a major fungal disease 60 years after the discovery of the pathogen. Mycopathologia. 2019;184(6):709-20. https://doi.org/10.1007/S11046-01900410-2.

20. Li X, Hu W, Wan Q, Lei Q, Sun C, Hou Z, et al. Non-Hiv talaromycosis: radiological and clinical analysis. Medicine (Baltimore). 2020;99(10):E19185. https://doi.org/10.1097/ Md.0000000000019185.

21. Hjf Salzer, Burchard G, Oa Cornely, Lange C, Rolling T, Schmiedel S, et al. Diagnosis and management of systemic endemic mycoses causing pulmonary disease. Respiration. 2018;96(3):283-301. https://doi.org/10.1159/000489501.

22. Qiu Y, Jq Zhang, Ml Pan, Zeng W, Tang Sd, Cm Tan. Determinants of prognosis in talaromyces marneffei infections with respiratory system lesions. Chin Med J (Engl). 2019;132(16):1909-18. https://doi.org/10.1097/Cm9. 0000000000000345.

23. Boonsarngsuk V, Eksombatchai D, Kanoksil W, Tantrakul V. Airway obstruction caused by penicilliosis: a case report and review of the literature. Arch Bronconeumol. 2015;51(5):E25-8. https://doi.org/10.1016/J.Arbres.2014. 04.015 .

24. Qiu Y, Pan M, Zhang J, Zhong X, Li Y, Zhang H, et al. Two Unusual cases of human immunodeficiency virus-negative patients with talaromyces marneffei infection. Am J Trop Med Hyg. 2016;95(2):426-30. https://doi.org/10.4269/ Ajtmh.15-0789.

25. Qiu Y, Tang Y, Zhang J, Yi X, Zhong X, Liu G, et al. A retrospective analysis of seven patients with acquired immunodeficiency syndrome and pharyngeal and/or laryngeal talaromyces marneffei infection. Clin Otolaryngol. 2017;42(5):1061-6. https://doi.org/10.1111/Coa.12838.

26. Wongkamhla T, Chongtrakool P, Jitmuang A. A case report of talaromyces marneffei oro-pharyngo-laryngitis: a rare manifestation of talaromycosis. Bmc Infect Dis. 2019;19(1):1034. https://doi.org/10.1186/S12879-0194650-7. 
27. Singh A, Atallah S, Al-Shyoukh A, Dacunha M, Mizusawa M. Localized talaromyces marneffei infection presenting as a tonsillar mass mimicking malignancy. Idcases. 2020;21:E00824. https://doi.org/10.1016/J.Idcr.2020. E00824.

28. Nittayananta W. Penicilliosis marneffei: another aids defining illness in Southeast Asia. Oral Dis. 1999;5(4):286-93. https://doi.org/10.1111/J.1601-0825. 1999.Tb00091.X.

29. Sa Joosten, Hannan L, Heroit G, Boerner E, Irving L. Penicillium marneffei presenting as an obstructing endobronchial lesion in an immunocompetent host. Eur Respir J. 2012;39(6):1540-3. https://doi.org/10.1183/09031936. 00156911.

30. Mcshane H, Cm Tang, Cp Conlon. Disseminated penicillium marneffei infection presenting as a right upper lobe mass in an Hiv positive patient. Thorax. 1998;53(10):905-6. https://doi.org/10.1136/Thx.53.10.905.

31. Hsu Jh, Ms L, Dai Zk Wu, Chiou SS. Life-threatening airway obstruction caused by penicilliosis in a leukemic patient. Ann Hematol. 2009;88(4):393-5. https://doi.org/ 10.1007/S00277-008-0606-5.

32. Yg Wang, Jm Cheng, Hb Ding, Lin X, Chen Gh, Zhou M, et al. Study on the clinical features and prognosis of penicilliosis marneffei without human immunodeficiency virus infection. Mycopathologia. 2018;183(3):551-8. https://doi. org/10.1007/S11046-017-0236-3.

33. Nishikubo M, Doi A, Takegawa H, Yamashita D, Ohira J, Nishioka H. Asymptomatic pulmonary penicilliosis with a lung mass in an hiv-infected patient. J Gen Fam Med. 2020;21(4):152-4. https://doi.org/10.1002/Jgf2.325.

34. Supparatpinyo K, Khamwan C, Baosoung V, Ke Nelson, Sirisanthana T. Disseminated penicillium marneffei infection in Southeast Asia. Lancet. 1994;344(8915):110-3. https://doi.org/10.1016/S0140-6736(94)91287-4.

35. Qiu Y, Zhang J, Liu G, Zhong X, Deng J, He Z, et al. Retrospective analysis of 14 cases of disseminated penicillium marneffei infection with osteolytic lesions. Bmc Infect Dis. 2015;15:47. https://doi.org/10.1186/S12879015-0782-6.

36. Cy L, Hy S, My C, Sm H, Sheng Wh, Yc Lo, et al. Aetiology of cavitary lung lesions in patients with Hiv infection. Hiv Med. 2009;10(3):191-8. https://doi.org/10.1111/J.14681293.2008.00674.X.

37. Qiu Y, Zeng W, Zhang H, Zhong X, Tang S, Zhang J. Comparison of pleural effusion features and biomarkers between talaromycosis and tuberculosis in non-human immunodeficiency virus-infected patients. Bmc Infect Dis. 2019;19(1):745. https://doi.org/10.1186/S12879-019-43766.

38. Lin F, Qiu Y, Zeng W, Liang Y, Zhang J. Talaromyces marneffei infection in a lung cancer patient: a rare case report. Bmc Infect Dis. 2019;19(1):336. https://doi.org/10. 1186/S12879-019-3968-5.

39. Darma S, Saleh Yd, Wibawa T. Lung talaromyces marneffei infection in an indonesian papillary thyroid carcinoma patient. Sage Open Med Case Rep. 2017;5:2050313x17744918. https://doi.org/10.1177/ $2050313 \times 17744918$.

40. Zhang J, Huang X, Zhang X, Zhu Y, Liao K, Ma J, et al. Coinfection of disseminated talaromyces marneffei and mycobacteria kansasii in a patient with papillary thyroid cancer: a case report. Medicine (Baltimore). 2017;96(52):E9072. https://doi.org/10.1097/Md. 0000000000009072.

41. De Monte A, Risso K, Ac Normand, Boyer G, L'ollivier C, Marty $\mathrm{P}$, et al. chronic pulmonary penicilliosis due to penicillium marneffei: late presentation in a french traveler. J Travel Med. 2014;21(4):292-4. https://doi.org/10.1111/ Jtm. 12125 .

42. As Sekhon, Stein L, Ak Garg, Wa Black, Glezos Jd, Wong C. Pulmonary penicilliosis marneffei: report of the first imported case in Canada. Mycopathologia. 1994;128(1):3-7. https://doi.org/10.1007/Bf01104271.

43. Wang $\mathrm{Ph}, \mathrm{Hc} \mathrm{W}$, Liao $\mathrm{Ch}$. disseminated penicillium marneffei mimicking paradoxical response and relapse in a nonHiv patient with pulmonary tuberculosis. J Chin Med Assoc. 2015;78(4):258-60. https://doi.org/10.1016/J.Jcma.2013. 11.009.

44. Yu X, Cai X, Xu X, Zhang L, Huang X, Wang L, et al. Fungemia caused by penicillium marneffei in an immunocompetent patient with copd: a unique case report. Medicine (Baltimore). 2018;97(3):E9658. https://doi.org/10.1097/ Md.0000000000009658.

45. Ntm T, Jfw C, Vt Ly, Ht N, Hta H, Nph L, et al. Superiority of a novel mplp antigen detection enzyme immunoassay compared to standard bactec blood culture in the diagnosis of talaromycosis. Clin Infect Dis. 2020. https://doi.org/10. 1093/Cid/Ciaa826.

46. Le T, Cash-Goldwasser S, Pv Tho, Np Lan, Ji Campbell, Hr Van Doorn, et al. Diagnosing rhodococcus equi infections in a setting where tuberculosis is highly endemic: a double challenge. J Clin Microbiol. 2015;53(4):1431-3. https://doi. org/10.1128/Jcm.02284-14.

47. Xj Han, Dh Su, Jy Yi, Yw Zou, Y1 Shi. a literature review of blood-disseminated p. marneffei infection and a case study of this infection in an Hiv-negative child with comorbid eosinophilia. Mycopathologia. 2019;184(1):129-39. https:// doi.org/10.1007/S11046-018-0255-8.

48. Vanittanakom N, Cooper CR, Fisher MC, Sirisanthana T. Penicillium marneffei infection and recent advances in the epidemiology and molecular biology aspects. Clin Microbiol Rev. 2006;19(1):95-110. https://doi.org/10.1128/Cmr. 19.1.95-110.2006.

49. Supparatpinyo K, Chiewchanvit S, Hirunsri P, Uthammachai C, Ke Nelson, Sirisanthana T. Penicillium marneffei infection in patients infected with human immunodeficiency virus. Clin Infect Dis. 1992;14(4):871-4. https://doi.org/10. 1093/Clinids/14.4.871.

50. Zheng J, Gui X, Cao Q, Yang R, Yan Y, Deng L, et al. A Clinical study of acquired immunodeficiency syndrome associated penicillium marneffei infection from a non-endemic area in China. PLoS ONE. 2015;10(6):E0130376. https://doi.org/10.1371/Journal.Pone.0130376.

51. Li X, Zheng Y, Wu F, Mo D, Liang G, Yan R, et al. Evaluation of quantitative real-time pcr and platelia galactomannan assays for the diagnosis of disseminated talaromyces marneffei infection. Med Mycol. 2020;58(2):181-6. https://doi.org/10.1093/Mmy/Myz052.

52. Yoshimura Y, Sakamoto Y, Lee K, Amano Y, Tachikawa N. Penicillium marneffei infection with B-D-glucan elevation: a case report and literature review. Intern Med. 
2016;55(17):2503-6.

Internalmedicine.55.6173.

https://doi.org/10.2169/

53. Yf Wang, Jp Cai, Wang Yd, Dong H, Hao W, Lx Jiang, et al. Immunoassays based on penicillium marneffei Mp1p derived from pichia pastoris expression system for diagnosis of penicilliosis. Plos One. 2011;6(12):E28796. https:// doi.org/10.1371/Journal.Pone.0028796.

54. Prakit K, Nosanchuk Jd, Pruksaphon K, Vanittanakom N, Youngchim S. A novel inhibition elisa for the detection and monitoring of penicillium marneffei antigen in human serum. Eur J Clin Microbiol Infect Dis. 2016;35(4):647-56. https://doi.org/10.1007/S10096-016-2583-2.

55. Pruksaphon K, Intaramat A, Ratanabanangkoon K, Nosanchuk Jd, Vanittanakom N, Youngchim S. Development and characterization of an immunochromatographic test for the rapid diagnosis of talaromyces (penicillium) marneffei. Plos One. 2018;13(4):E0195596. https://doi.org/10.1371/ Journal.Pone.0195596.

56. Le T, Nv Kinh, Ntk Cuc, Nln Tung, Nt Lam, Ptt Thuy, et al. A trial of itraconazole or amphotericin B for Hiv-associated talaromycosis. N Engl J Med. 2017;376(24):2329-40. https://doi.org/10.1056/Nejmoa1613306.

57. Wang P, Chen Y, Xu H, Ding L, Wu Z, Xu Z, et al. Acute disseminated talaromyces marneffei in an immunocompetent patient. Mycopathologia. 2017;182(7-8):751-4. https://doi.org/10.1007/S11046-017-0127-7.
58. Qiu Y, Lu D, Zhang J, Zhong X, Liu G, Li B. Treatment of disseminated talaromyces marneffei with tracheal infection: two case reports. Mycopathologia. 2015;180(3-4):245-9. https://doi.org/10.1007/S11046-015-9891-4.

59. Zhang W, Ye J, Qiu C, Wang L, Jin W, Jiang C, et al. rapid and precise diagnosis of $t$ marneffei pulmonary infection in a Hiv-negative patient with autosomal-dominant stat3 mutation a case report. Ther Adv Respir Dis. 2020;14:1753466620929225. https://doi.org/10.1177/ 1753466620929225.

60. Li Hr Xu, Nl Lin M, Xl Hu, Chen Jh, Ys Chen, et al. Diffuse interstitial and multiple cavitary lung lesions due to talaromyces marneffei infection in a non-Hiv patient. New Microbes New Infect. 2015;8:14-6. https://doi.org/10.1016/ J.Nmni.2015.06.010.

61. Furusawa H, Miyazaki Y, Sonoda S, Tsuchiya K, Yaguchi T, Kamei K, et al. Penicilliosis marneffei complicated with interstitial pneumonia. Intern Med. 2014;53(4):321-3. https://doi.org/10.2169/Internalmedicine.53.1465.

Publisher's Note Springer Nature remains neutral with regard to jurisdictional claims in published maps and institutional affiliations. 